\title{
T helper 17 cells are involved in the local and systemic inflammatory response in community-acquired pneumonia
}

\author{
Marthe S Paats, ${ }^{1}$ Ingrid M Bergen, ${ }^{1}$ Wessel E J J Hanselaar, ${ }^{2}$ \\ E Christine Groeninx van Zoelen, ${ }^{3}$ Henry A Verbrugh, ${ }^{4}$ Henk C Hoogsteden, ${ }^{1}$ \\ Bernt van den Blink, ${ }^{1}$ Rudi W Hendriks, ${ }^{1}$ Menno M van der Eerden ${ }^{1}$
}

\begin{abstract}
- Additional material is published online only. To view please visit the journal online (http://dx.doi.org/10.1136/ thoraxjnl-2012-202168).

${ }^{1}$ Department of Pulmonary Medicine, Erasmus MC Rotterdam, Rotterdam, The Netherlands

2Department of Pulmonary Medicine, Sint Franciscus Gasthuis, Rotterdam, The Netherlands

${ }^{3}$ Department of Intensive Care, Erasmus MC Rotterdam, Rotterdam, The Netherlands ${ }^{4}$ Department of Medical Microbiology, Erasmus MC Rotterdam, Rotterdam, The Netherlands
\end{abstract}

Correspondence to Dr Menno van der Eerden, Department of Pulmonary Medicine, Erasmus MC, 'sGravendijkwal 230, Rotterdam 3015 CE, The Netherlands; $m$. vandereerden@erasmusmc. nIRWH and MME contributed equally.

Received 17 May 2012 Revised 19 November 2012 Accepted 14 December 2012 Published Online First 12 January 2013

\footnotetext{
To cite: Paats MS Bergen IM, Hanselaar WEJJ, et al. Thorax 2013;68: 468-474.
}

\begin{abstract}
Background Recent findings in mouse models suggest that T helper (Th)17 cells, characterised by production of interleukin (IL)-17A and IL-22, are involved in the immunopathogenesis of pneumonia.

Objective In this study, we aimed to identify the involvement of Th17 cells in human community-acquired pneumonia (CAP).

Design Within $24 \mathrm{~h}$ of admission, T cells from peripheral blood $(n=39)$ and bronchoalveolar lavage $(B A L, n=20)$ of CAP patients and of 10 healthy individuals were analysed by intracellular flow cytometry for the production of various cytokines, including IL-17A and IL-22. Peripheral blood T cells were also analysed 7 and 30 days after admission. Th17 cytokine profiles were correlated with pneumonia severity index and microbial aetiology.

Results In the BAL of CAP patients, proportions of IL17A and IL-22 single positive, as well as IL-17A/IL-22

\section{Key messages}

What is the key question?

- Are Thelper (Th)17 cells involved in the pathogenesis of community-acquired pneumonia (CAP)?

\section{What is the Bottom line?}

- Th17 cells, and especially IL-17A/IL-22 double positive Th17 cells, are engaged in the local and systemic immune response in human pneumonia.

\section{Why read on?}

- This study is the first to demonstrate the Th17 response as a prominent feature of CAP in humans.
\end{abstract} double positive CD4 T cells were significantly increased compared with healthy individuals. Significantly increased proportions of IL-17A/IL-22 double positive CD4 T cells in BAL were found in non-severe and severe CAP patients, as well as in pneumococcal and nonpneumococcal CAP. In the peripheral blood of CAP patients upon admission, we found significantly increased proportions of IL-17A/IL-22 double positive CD4 T cells. One week after admission, the proportions of these double positive cells were still significantly increased in CAP patients compared with healthy individuals.

Conclusions These data indicate that Th17 cells are engaged in the local and systemic immune response in human pneumonia. Especially, IL-17A/IL-22 double positive Th17 cells may be involved in the immunopathogenesis of CAP.

\section{INTRODUCTION}

Community-acquired pneumonia (CAP) is a common and serious illness despite the use of pathogen-directed or empirical broad-spectrum antimicrobial therapy. ${ }^{1}$ Major gaps remain in the understanding of its pathogenesis; it is not clear why some individuals can easily control bacterial challenges and remain healthy, whereas others develop pneumonia.
The immunopathology of CAP is characterised by a strong local and systemic acute-phase response. Several components of the innate immune system, such as neutrophils and macrophages, have been identified as key mediators of bacterial clearance in the lungs. ${ }^{2}{ }^{3}$ The role of T cells in the pathogenesis of pneumonia is, however, less evident. HIV patients with depleted CD4 T cells are at increased risk for developing lung infections, suggesting that these cells are involved in the immune defence against pneumonia. ${ }^{4}$

Recently, it has been demonstrated that the novel $\mathrm{T}$ helper (Th)17 subset expressing the signature cytokines interleukin (IL)-17A and IL-22, has a protective role in host defence against different respiratory pathogens in the mouse. ${ }^{5}$ IL-17A is thought to promote chemokine and proinflammatory cytokine production, and consequent recruitment and activation of neutrophils. ${ }^{6}$ IL-22 controls tissue responses during inflammation, for instance, by increasing lung epithelial proliferation and transepithelial resistance to injury. ${ }^{7} 8$ Several groups showed that IL-17 mediates pneumococcal immunity in mice, ${ }^{9-11}$ and that acquired immunity to pneumococcal colonisation is associated with the Th17 subset. $^{9}{ }^{11}$ Moreover, IL-17A increased pneumococcal killing by human neutrophils both in the absence and in the presence of antibodies 
and complement. ${ }^{9}$ In mouse models of Klebsiella pneumoniae infection, both IL-17A and IL-22 production were required for optimal bacterial clearance. ${ }^{7} 12 \quad 13$ Th17 responses are also involved in murine host defence against other bacterial pathogens including Mycoplasma pneumoniae, ${ }^{14}$ Bordetella pertussis $^{15}$ and Pseudomonas aeruginosa, ${ }^{16}$ as well as respiratory viruses. ${ }^{17} 18$ Human studies on Th17 cells in pneumonia are currently lacking, except for the demonstration of a role for Th17 cells in clearing pulmonary infections in the hyper IgE-syndrome. In this disease, caused by mutations in STAT3, Th17, cells are lost and patients manifest chronic, recurrent and severe bacterial and fungal infections. ${ }^{19}$

In conclusion, whereas there is accumulating evidence for the involvement of Th17 cells in bacterial and viral respiratory infection in mice, in humans, Th17 cells are largely unexplored. In this study, we therefore aimed to investigate the presence of Th17 cells both locally and systemically in patients with CAP and to correlate these findings with disease severity and causative pathogen.

\section{METHODS}

\section{Subjects}

A prospective study was performed between January 2009 and May 2011. Thirty-nine CAP patients admitted through the emergency ward of the Erasmus MC and the Sint Franciscus Gasthuis, both teaching hospitals, were enrolled in the study. The medical ethics committees of both hospitals approved of the study (MEC 2008-323 Erasmus MC Rotterdam and Sint Franciscus Gasthuis Rotterdam, The Netherlands). Written informed consent was obtained from patients or closest relatives.

Patients who fulfilled the following criteria were included: (1) age $\geq 18$ years; (2) clinical presentation of an acute illness with one or more of the following symptoms suggesting CAP: presence of fever $\left(t \geq 38^{\circ} \mathrm{C}\right)$, dyspnoea, coughing (with or without expectoration of sputum), chest pain and (3) presence of new consolidation(s) on the chest radiograph. Patients were excluded if one of the following criteria applied: nursing home residency or hospital admission in the previous 15 days, presence of immunosuppression (eg, HIV infection, systemic immunosuppressive agents including oral corticosteroids and chemotherapy), presence of a systemic autoimmune disease, pulmonary tuberculosis, bronchiectasis, malignancy or obstruction pneumonia. Ten healthy volunteers matched for age, sex and smoking status, and without a history of cardiac or pulmonary disease, malignancy or autoimmune disease served as controls.

\section{Cell collection and isolation}

After written informed consent, and within $24 \mathrm{~h}$ after hospital admission, venous blood samples were collected $(n=39)$. In a subset of patients $(n=20)$ and in all healthy individuals $(n=10)$, a bronchoalveolar lavage (BAL) was performed with a flexible fibreoptic bronchoscope (Olympus) according to recommended guidelines. ${ }^{20}$ The bronchoscope was wedged into a subsegmental bronchus corresponding to the area of the most dense consolidation on the chest radiograph. In patients with diffuse pulmonary infiltrates and healthy individuals, the middle lobe or lingular division was chosen. The first sample of BAL, which was obtained after instilling and aspirating $20 \mathrm{ml}$ of sterile saline was used for microbiological analysis. Next, three aliquots of $20 \mathrm{ml}$ sterile saline each was instilled and gently aspirated for

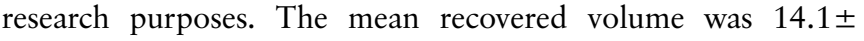
$9.6 \mathrm{ml}$. Venous blood samples were collected directly prior to the BAL procedure. At days 7 and 30 after admission, additional venous blood samples were collected.

BAL and peripheral blood mononuclear cells were isolated and stored as described previously. ${ }^{21}$

\section{Flow cytometry analysis}

For cytokine analysis, cells were stimulated with ionomycine (Sigma) and phorbol 12-myristate 13-acetate (PMA) (Sigma) in the presence of Brefeldin A (ebiosciences) and incubated for $4 \mathrm{~h}$ at $37^{\circ} \mathrm{C}$. Cells were stained as described previously ${ }^{22}$ and measured on a LSRII Flowcytometer (BD Biosciences). Data were analysed using FlowJo (BD) software. Isotype controls and fluorescence minus one control were used to set gates. Flow cytometry experiments of all samples were performed in a single batch in order to eliminate the influence of day-to-day variation.

\section{Microbiological studies}

Prior to initiation of antibiotic therapy, two sets of blood cultures were taken from each patient. In patients with a productive cough, sputum samples were obtained upon admission for Gram staining and culture. Furthermore, urine samples were taken for antigen detection of $S$ pneumoniae and Legionella pneumophila serogroup 1. Gram stainings and cultures were performed on BAL specimens and, if clinical symptoms suggested, pharyngeal swabs were taken for identification of viral pathogens.

\section{Statistical analysis}

Data are shown as mean (SD) in case of normally distributed data or median with IQR or percentiles when not normally distributed. Most data were not normally distributed and, therefore, non-parametric tests were used to compare groups (Kruskal-Wallis test for across-group comparison of three or more groups, Mann-Whitney U test for pairwise analyses). Normally distributed data were analysed by unpaired t tests. Data analysis was performed using Statistical Package for Social Sciences (SPSS) 17.0 and Prism 5.01 (GraphPad). For statistical significance, $\mathrm{p}<0.05$.

\section{RESULTS}

\section{Clinical characteristics of study population}

Venous blood samples were drawn from all 39 CAP patients included in the study; in 20 patients also a BAL was performed. No significant differences in clinical parameters were present between the total patient group and the subset of patients undergoing BAL procedure (table 1). Based on pneumonia severity index (PSI) scores, patients were allocated to the nonsevere (PSI class I-III, $\mathrm{n}=22(56 \%)$ ) or severe (PSI class IV-V, $\mathrm{n}=17(44 \%))$ CAP group. In 10 patients (26\%) $S$ pneumoniae was isolated. Thirteen patients (33\%) had a known nonpneumococcal bacterial $(n=10)$ or viral $(n=3)$ aetiology. In 16 patients (41\%), no causative pathogen could be identified (table 1). Patients with a pneumococcal pneumonia were not more severely ill than patients with a bacterial non-pneumococcal aetiology (see online supplementary table S1). For the distribution of identified pathogens: see online supplementary table S2.

As a control group, we included 10 healthy individuals matched for age (54.8 \pm 5.7 years), gender (six males) and smoking status (four smokers). 
Table 1 Baseline characteristics of CAP patients

\begin{tabular}{|c|c|c|}
\hline & PBMC $(n=39)$ & BAL $(n=20)$ \\
\hline Mean (SD) age (years) & $55.6 \pm 18.5$ & $60.6 \pm 19.0$ \\
\hline Male sex, $\mathrm{n}(\%)$ & $25(64)$ & $13(65)$ \\
\hline Smoking, n (\%) & $15(38)$ & $8(40)$ \\
\hline \multicolumn{3}{|l|}{ Comorbidity, n (\%) } \\
\hline COPD & $10(26)$ & $5(25)$ \\
\hline Heart disease & $8(21)$ & $6(30)$ \\
\hline Neurological disorder & $6(15)$ & $4(20)$ \\
\hline Chronic renal disease & $1(3)$ & $1(5)$ \\
\hline Diabetes mellitus & $6(15)$ & $2(10)$ \\
\hline \multicolumn{3}{|l|}{ Pneumonia Severity Index, n (\%) } \\
\hline Low I & $10(26)$ & $3(15)$ \\
\hline Low II & $7(18)$ & $5(25)$ \\
\hline Low III & $5(13)$ & $2(10)$ \\
\hline Moderate IV & $12(31)$ & $6(30)$ \\
\hline High V & $5(13)$ & $4(20)$ \\
\hline Mean \pm SD CRP (mg/l) & $207 \pm 151$ & $219 \pm 169$ \\
\hline \multicolumn{3}{|l|}{ Chest consolidation(s), $\mathrm{n}(\%)$} \\
\hline Unilobar, unilateral & $32(82)$ & $16(80)$ \\
\hline Multilobar, unilateral & $5(13)$ & $2(10)$ \\
\hline Bilateral & $2(5)$ & $2(10)$ \\
\hline ICU admission & $8(21)$ & $5(25)$ \\
\hline Mechanical ventilation, n (\%) & $6(15)$ & $4(20)$ \\
\hline Bacteraemia, n (\%) & $6(15)$ & $5(25)$ \\
\hline Hypoxaemia (sat<90\%), n (\%) & $13(33)$ & $7(35)$ \\
\hline Mean \pm SD LOS (days) & $10 \pm 14$ & $13 \pm 18$ \\
\hline Mortality, n (\%) & $2(5)$ & $2(10)$ \\
\hline \multicolumn{3}{|l|}{ Microbiological species, n (\%) } \\
\hline Streptococcus pneumoniae & $10(26)$ & 7 (35) \\
\hline Other bacterial & $10(26)$ & $5(25)$ \\
\hline Viral & $3(7.7)$ & $2(10)$ \\
\hline Unknown & $16(41)$ & $6(30)$ \\
\hline
\end{tabular}

BAL, bronchoalveolar lavage; CAP, community-acquired pneumonia; COPD, chronic obstructive pulmonary disease; PBMC, peripheral blood mononuclear cells; LOS, length of hospital stay. For all clinical parameters $p>0.35$.

\section{Comparison of cytokine expression between CAP patients and healthy individuals}

First, we quantified CD4, CD8 and $\gamma \delta \mathrm{T}$ cells in peripheral blood and BAL of all study subjects using flow cytometry. No significant differences were found in proportions of these lymphocyte subsets between CAP patients and healthy individuals (see online supplementary table S3). Absolute numbers of CD4 T cells in peripheral blood were similar in CAP patients upon admission compared with day 7 after admission (see online supplementary figure S1).

Next, we activated total peripheral blood mononuclear cell fractions and BAL cell suspensions and determined proportions of intracellular IL-17A and IL-22 single and double positive CD4 T cells (figure 1A), as well as proportions of IFN (Th1) and IL-4 (Th2) positive CD4 T cells in peripheral blood (data not shown).

Based on PSI scores upon admission, CAP patients were distributed over a non-severe $(n=22)$ and a severe patient group $(\mathrm{n}=17)$. In peripheral blood, no differences in the proportions of IL-17A and IL-22 single positive CD4 T cells were found between CAP patients and healthy individuals. The proportions of IL-17A/IL-22 double positive CD4 T cells were, however, significantly higher in the total CAP patient group and in severe CAP patients compared with healthy individuals (figure 1B). In BAL of CAP patients, we found significantly increased proportions of IL-17A and IL-22 single and double positive $\mathrm{CD} 4 \mathrm{~T}$ cells (both non-severe and severe patients, when compared with healthy individuals; figure 1C). No significant differences were found in the proportions of IFN $\gamma$ or IL-4 single positive or IL-17A/IFN $\gamma$ double positive CD4 T cells in blood between CAP patients and healthy individuals (data not shown).

Proportions of IL-17A and IL-22 single and double positive CD4 T cells were significantly higher in BAL when compared with peripheral blood (all $\mathrm{p}<0.001$; figure 1 ). However, no correlations were found between IL-17A and IL-22-positive CD4 T cells in blood and BAL of CAP patients (data not shown).

Moreover, we determined levels of IL-17 and IL-22 in serum and BAL fluid by ELISA. Levels of IL-17 were below detection in both compartments. ${ }^{22}$ IL-22 levels in serum, but not in BAL fluid, were significantly higher in CAP patients compared with healthy individuals (see online supplementary figure S3). No correlations were found between IL- $17^{+}$, IL- $22^{+}$or IL17/IL-22 double positive CD4 T cells in blood or BAL and levels of IL-22 in serum or BAL fluid (data not shown).

We also correlated Th17 responses in blood and BAL with other clinical parameters. Proportions of IL-17A/IL-22 double positive CD4 $\mathrm{T}$ cells in peripheral blood upon admission of CAP patients admitted to the Intensive Care Unit (ICU) were significantly higher compared with the non-ICU patient group $(\mathrm{p}=0.03$; see online supplementary figure $\mathrm{S} 2)$. No other correlations were found between Th17 responses and parameters of disease severity including PSI score, need for mechanical ventilation, extent of pulmonary consolidations on chest x-ray, bacteraemia, hypoxaemia, c-reactive protein (CRP) levels or mortality. The time of onset of symptoms before hospital admission, the use of antibiotics previous to hospital admission or the length of hospital admission also did not correlate with Th17 responses in blood or BAL of CAP patients (data not shown).

In addition to CD4 T cells, IL-17A and IL-22 can also be produced by other T-cell subsets, including $\gamma \delta$ and CD8 T cells. ${ }^{23}$ The proportions of IL- $17 \mathrm{~A}^{+}$or IL- $22^{+} \gamma \delta$ T cells in peripheral blood and BAL were very low and did not differ between CAP patients and healthy individuals. Likewise, the proportions of cytokine positive CD8 T cells in peripheral blood were not significantly increased in patients compared with healthy individuals. In BAL, the proportions of IL-17A and IL-22 single and double positive CD8 $\mathrm{T}$ cells were significantly elevated in patients when compared with healthy individuals, but percentages were 5-10-fold lower than those in the CD4 T cell compartment (data not shown).

\section{Correlation of Th17 cells with microbiological aetiology}

In the total CAP patient group, $10(26 \%)$ patients had $S$ pneumoniae isolated as the causative pathogen. Next, we compared patients with a pneumococcal pneumonia with patients with a known bacterial causative pathogen other than $S$ pneumoniae (see online supplementary table S1). CAP patients with a pneumococcal aetiology were not sicker than patients presenting with CAP of another aetiology. Furthermore, cytokine profiles of peripheral blood CD4 T cells were not significantly different between pneumococcal pneumonia, bacterial non-pneumococcal pneumonia (NP), and healthy individuals (figure 2A). By contrast, both patients with pneumococcal pneumonia and patients with a bacterial non-pneumococcal pathogen isolated, had significantly increased proportions of IL-17A and IL-22 single and double positive CD4 T cells in BAL, compared with healthy individuals (figure 2B). Consistent with the heterogeneity of pathogens involved, non-pneumococcal patients showed a large range in the proportions of cytokine expressing CD4 T cells, 
A
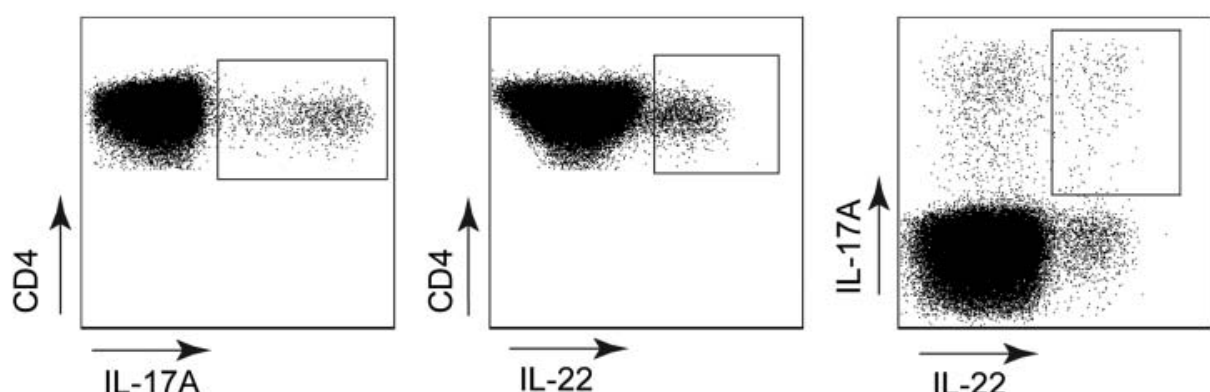

B PBMC

IL-17A

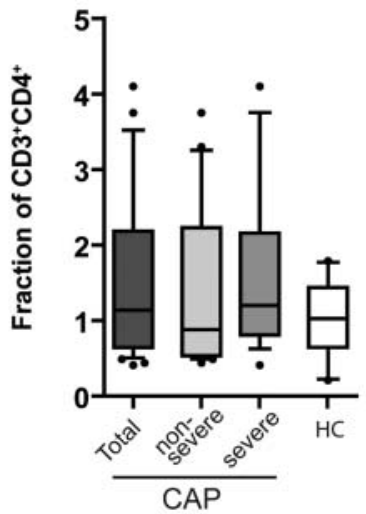

C BAL

IL-17A

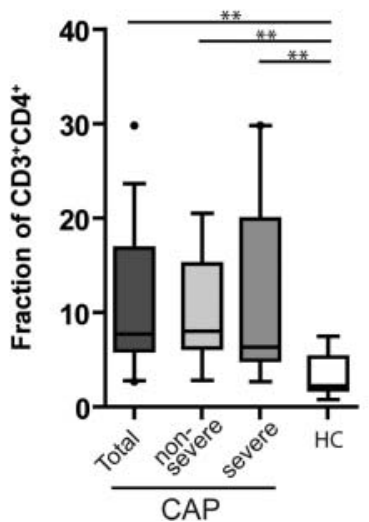

IL-22

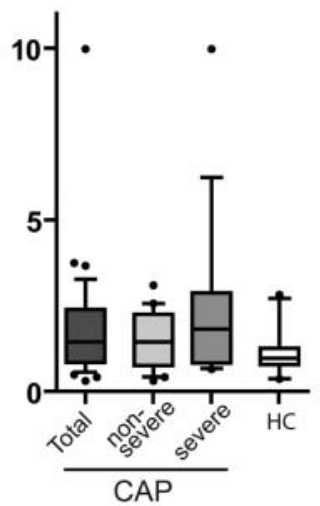

IL-22

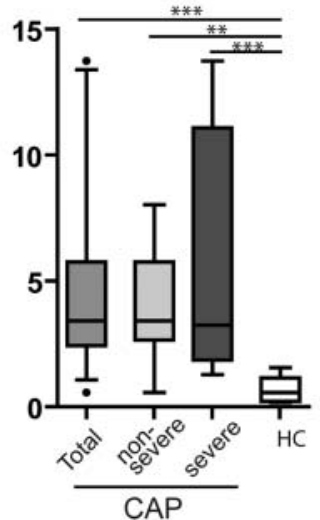

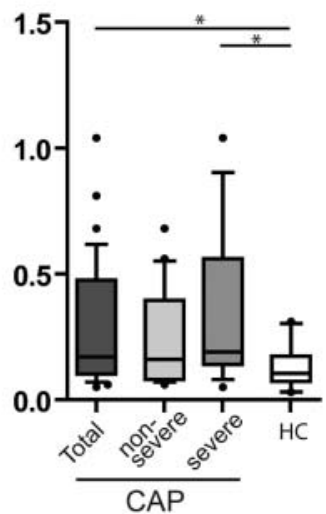

IL-17A/IL-22

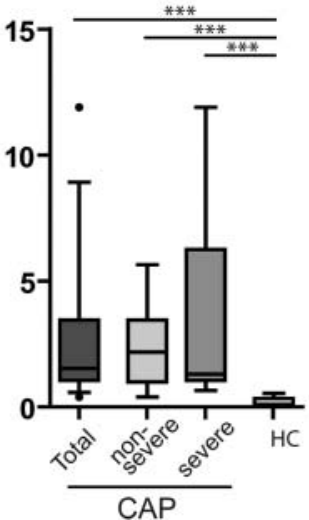

IL-17A/L-22

Figure 1 Cytokine expression by CD4 T cells in peripheral blood and BAL of CAP patients and healthy individuals. CAP patients were under divided as non-severe CAP (PSI class I-III) or as severe CAP (PSI class IV-V). (A) Flow cytometric characterisation of CD4 T cells. CD4 T cells were analysed for IL-17A expression (left panel), IL-22 expression (middle panel) and double expression of IL-17A and IL-22 (right panel). PBMC were gated from live CD56- cells, BAL cells from live cells. Results are shown as dot plots. (B). Proportions of total CD4 T cells positive for the indicated cytokines in PBMC from the total group of CAP patients $(n=39)$, non-severe $(n=22)$ and severe $(n=17)$ CAP patients, versus healthy individuals $(n=10)$. (C) Proportions of total CD4 T cells positive for the indicated cytokines in BAL from the total group of CAP patients $(n=20)$, severe $(n=10)$ and non-severe $(n=10)$ CAP patients versus healthy individuals $(n=10)$. Data are shown as box and whisker plots with 10th and 90 th percentiles. Bold lines represent median values. Differences between CAP groups and HC were tested with the Mann-Whitney $U$ test. ${ }^{*}: p<0.05 ;{ }^{* *}: p<0.01$; ${ }^{* * *}$ : $p<0.001$. CAP, community-acquired pneumonia; HC, healthy control; PBMC, peripheral blood mononuclear cells; BAL, bronchoalveolar lavage.

both in peripheral blood and in BAL. Up to $30 \%$ of CD4 T cells in BAL of CAP patients with a bacterial non-pneumococcal aetiology were IL-17 positive (figure $2 \mathrm{~B}$ ).

\section{Systemic Th17 cytokine responses over time in CAP}

Peripheral blood samples were drawn from CAP patients upon admission and at days 7 and 30 after admission. To investigate systemic Th17 responses over time, we focused on proportions of IL-17A ${ }^{+} \mathrm{IL}-22^{+} \mathrm{CD} 4 \mathrm{~T}$ cells, which upon admission were significantly higher in CAP patients than in healthy controls (figure 1B). At day 7 , these proportions were still significantly higher than those found in healthy individuals, and showed a small but not significant increase compared with values upon admission (figure $3 \mathrm{~A}$ ). At day 30 , proportions of $\mathrm{IL}-17 \mathrm{~A}^{+} \mathrm{IL}-22^{+} \mathrm{CD} 4$ 
A

IL-17A

IL-22

IL17/IL22
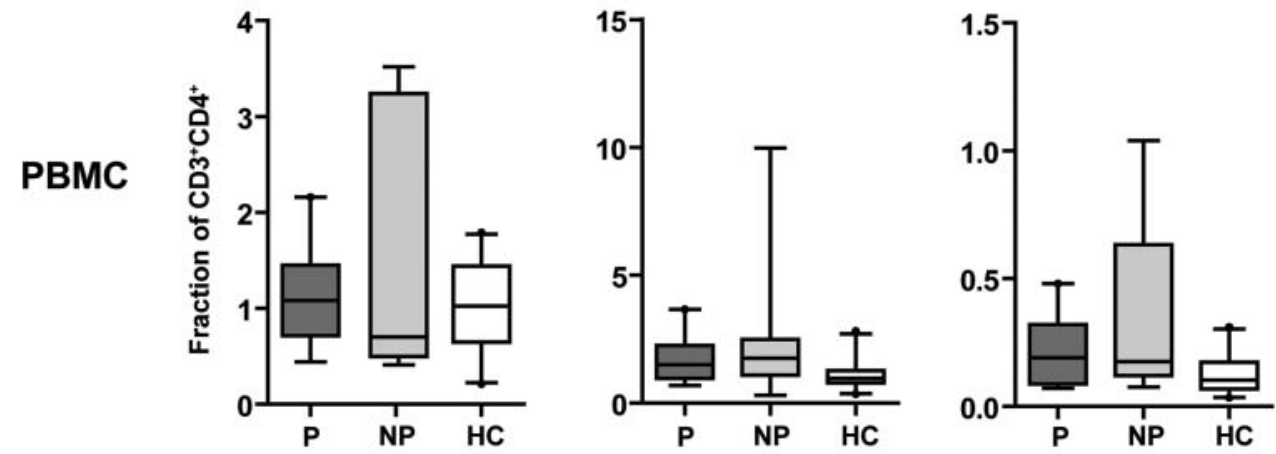

B
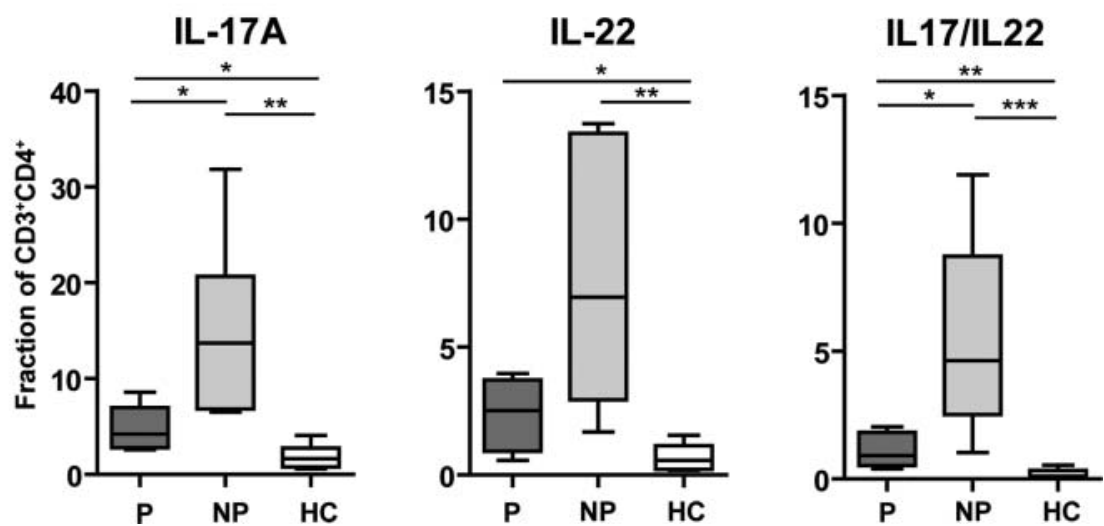

Figure 2 Cytokine expression by CD4 T cells in peripheral blood and BAL of community-acquired pneumonia (CAP) patients with pneumococcal pneumonia, bacterial non-pneumococcal pneumonia (NP) and healthy individuals. CAP patients were classified as having pneumococcal pneumonia (P) when Streptococcus pneumoniae was identified as the pathogenic micro-organism. NP patients had a bacterial pathogen isolated other that $S$ pneumoniae. (A) Proportions of total CD4 T cells positive for the indicated cytokines in PBMC from P $(n=10), N P(n=10)$ and HC $(n=10)$. (B) Proportions of total CD4 T cells positive for the indicated cytokines BAL from $P(n=7)$, NP $(n=5)$ and HC $(n=10)$. Data are shown as box and whisker plots with 10th and 90th percentiles. Bold lines represent median values. Differences between groups were tested with the Mann-Whitney $U$ test. ${ }^{*}: \mathrm{p}<0.05 ;{ }^{* *}: \mathrm{p}<0.01{ }^{* * *}: \mathrm{p}<0.001$. HC: healthy control. PBMC, peripheral blood mononuclear cells; BAL, bronchoalveolar lavage.

T cells in CAP patients were similar to those in healthy individuals (figure $3 \mathrm{~A}$ ).

Similar to the total group of CAP patients, also the group of severe CAP patients had significantly elevated proportions of IL-17A ${ }^{+} \mathrm{IL}-22^{+}$CD4 T cells upon admission and at day 7, when compared with healthy individuals (figure $3 \mathrm{~B}$ ). In non-severe CAP patients, the proportions of these cells were not significantly different from values in healthy individuals at any time point (figure 3B).

When comparing pneumococcal and bacterial nonpneumococcal CAP patients with healthy individuals at different time points, we found that patients with pneumococcal pneumonia only had significantly higher proportions of IL-17A ${ }^{+} \mathrm{IL}-22^{+}$CD4 $\mathrm{T}$ cells in their peripheral blood on day 7 after admission (figure $3 \mathrm{C}$ ).

\section{DISCUSSION}

In this study, we aimed to identify the involvement of Th17 cells in the immunopathogenesis of CAP. The most important findings are that, in the BAL of CAP patients, proportions of IL-17A and IL-22 single and double positive CD4 T cells were significantly increased compared with healthy individuals. Significantly increased proportions of IL-17A ${ }^{+} \mathrm{IL}-22^{+}$CD4 T cells in BAL were found in non-severe and severe CAP patients, as well as in pneumococcal and non-pneumococcal CAP. Upon admission, we found no differences in the proportions of IL-17A or IL-22 single positive CD4 T cells in peripheral blood between CAP patients and healthy individuals. The proportions of IL-17A ${ }^{+} \mathrm{IL}-22^{+}$CD4 T cells were, however, significantly elevated in the blood of CAP patients, and were still significantly increased 1 week after admission. Moreover, upon admission, proportions of IL-17A/IL-22 double positive CD4 T cells in peripheral blood of CAP patients admitted to the ICU were significantly higher than those in the non-ICU patient group.

While Th17 responses to pneumonia are well established in mice, ${ }^{7}{ }^{9-18}$ this is the first study demonstrating in vivo Th17 involvement as a distinct feature of CAP in humans. Our findings in peripheral blood point to an isolated Th17 response since we did not find increased proportions of circulating IFN $\gamma^{+}$ (Th1) or IL-4 ${ }^{+}$(Th2) T helper cells. An important difference between our study and the other studies on $\mathrm{T}$ cell cytokine responses in human pneumonia is the fact that we studied patients with a heterogeneous microbial aetiology. This could explain that, contradictory to our findings, previous studies showed Th1 or Th2 responses against certain respiratory pathogens. ${ }^{10}{ }^{17}{ }^{24-26}$ Kemp et al ${ }^{24}$ showed a Th1 response at the onset of pneumococcal infection. However, their study population also included patients without pneumonia. Furthermore, in 
Figure 3 Proportions of IL-17A ${ }^{+} \mathrm{IL}-22^{+}$double positive CD4 T cells in peripheral blood of community-acquired pneumonia (CAP) patients over time. (A). Proportions of IL-17A $\mathrm{A}^{+} \mathrm{IL}-22^{+} \mathrm{CD} 4 \mathrm{~T}$ cells in peripheral blood of CAP patients $(n=39)$ upon admission, at day 7 and day 30 after admission and in healthy individuals $(n=10)$. (B) Proportions of

IL-17A ${ }^{+} I \mathrm{~L}-22^{+}$CD4 T cells in peripheral blood of non-severe $(n=22)$ and severe CAP patients $(n=17)$ upon admission, at day 7 and day 30 after admission and in healthy individuals. (C). Proportions of IL-17A ${ }^{+}$IL-22 $2^{+}$CD4 T cells in peripheral blood of CAP patients with pneumococcal pneumonia $(n=10)$, bacterial non-pneumococcal pneumonia $(n=10)$ upon admission, at day 7 and day 30 after admission and in healthy individuals. Data are shown as box and whisker plots with 10th and 90th percentiles. Bold lines represent median values. *: Significantly different from HC $(p<0.05)$. HC, healthy control; NSCAP, non-severe community-acquired pneumonia; SCAP, severe community-acquired pneumonia.
A

IL-17A/IL-22

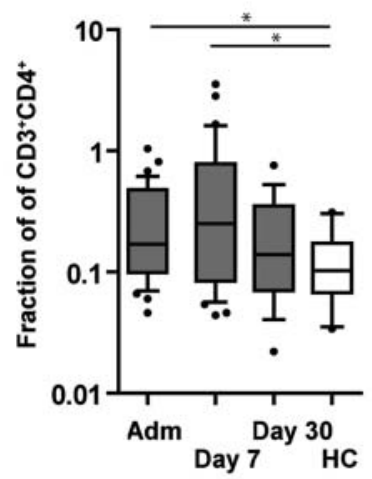

B

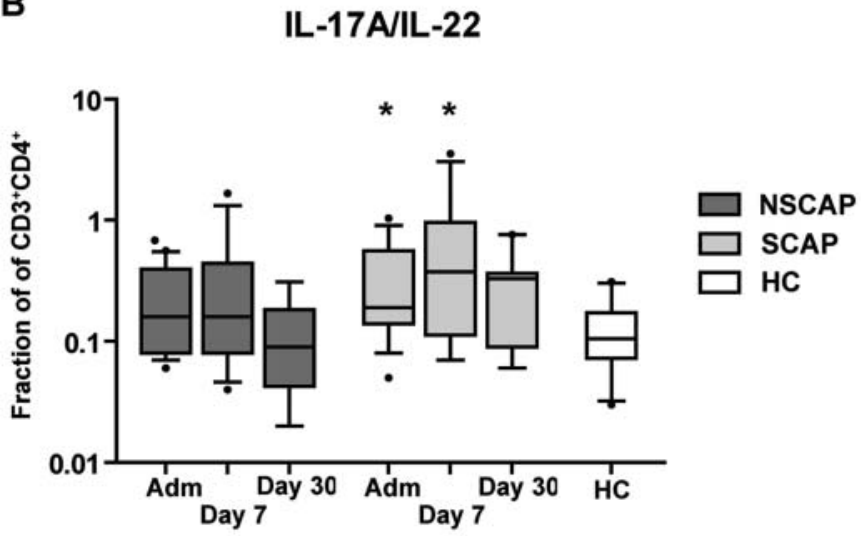

C

IL-17AIIL-22

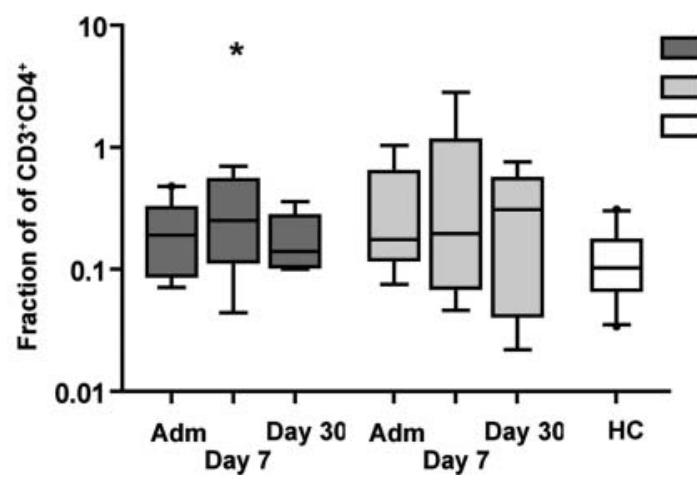

another study, human monocytes were in vitro infected with pneumococci, which resulted in both Th1 and Th17 responses. ${ }^{10}$ Patients with a pneumococcal aetiology had higher proportions of IL-17 and IL-22 single and double positive CD4 $\mathrm{T}$ cells in the BAL compared with healthy individuals. CAP patients with a bacterial non-pneumococcal aetiology, however, showed even higher proportions compared with the pneumococcal patient group, suggesting that the extent of Th17 involvement is pathogen-dependent. In pandemic 2009 H1N1 influenza, elevated plasma levels of both Th17 and Th1 related cytokines were observed, but the cellular source of these cytokines was not identified. ${ }^{17} 26$ The three patients in our study in whom a viral pathogen was isolated showed a wide range in cytokine expression, but IL-17A and IL-22 single and double positive CD4 $\mathrm{T}$ cells were, in all cases, above the median values of healthy individuals. A Th2 response was reported in Mycoplasma pneumonia. ${ }^{25}$ However, since we included only two patients with $M$ pneumoniae, we were not able to confirm these results.

It is conceivable that Th17 cytokines contribute to the vigorous recruitment and activation of neutrophils, which is one of the most important components of the innate immune response against bacterial infection. Particularly, since IL-17A has the ability to induce the expression of various proinflammatory mediators by epithelial and stromal cells, including IL-1, IL-6, TNF- $\alpha$, CXCL8, G-CSF and GM-CSF, all of which support recruitment or activation of neutrophils. ${ }^{27}$ In agreement with this notion, in a subgroup of patients for which peripheral blood neutrophil counts were available, we observed a positive correlation between the absolute numbers of circulating 
neutrophils and the proportions of $\mathrm{IL}-17^{+} \mathrm{CD} 4 \mathrm{~T}$ cells in BAL ( $\mathrm{p}=0.016, \mathrm{r}=0.73$; M.P. et al, unpublished).

In vitro differentiation of Th17 cells leads to cell populations with IL-17A or IL-22 single or double expression, a phenomenon also observed in vivo in the skin and lung. 2829 Coexpression of IL-17A and IL-22 is particularly of interest, as we observed high proportions of IL-17A/IL-22 doubleproducing $\mathrm{T}$ helper cells in both BAL and peripheral blood of CAP patients. IL-17A and IL-22 can act synergistically to augment the expression of genes involved in defence against microbial pathogens, such as $\beta$-defensin- 2 in keratinocytes. ${ }^{28}$ Additionally, in bleomycin-induced lung inflammation, IL-17A can regulate the expression, proinflammatory properties and tissue-protective functions of IL-22, thereby determining the functional consequences of IL-22 expression in the lung. ${ }^{29}$ Because of the protective effects of IL-17A and IL-22 in mouse models of pneumonia, ${ }^{7}{ }^{30}$ our finding of increased proportions of IL-17A ${ }^{+} \mathrm{IL}-22^{+} \mathrm{CD} 4 \mathrm{~T}$ cells in CAP supports the hypothesis that Th17 cells are necessary for a vital beneficial host response in human pneumonia.

Our results show increased IL-17A and IL-22 single positive CD4 $\mathrm{T}$ cells in BAL, but not in peripheral blood of CAP patients. Furthermore, CAP patients had higher proportions of IL-17A and, to a lesser extent, of IL-22 CD4 T cell in BAL than in peripheral blood, indicating that these cells preferentially home to, or are generated at the site of infection, for instance, the lungs.

Several factors have to be considered for the interpretation of our results. First of all, the number of patients included in this study is small, although comparable with other studies investigating local inflammatory responses. ${ }^{31}{ }^{32}$ Furthermore, like in other observational patient studies, there are uncontrolled factors present before patients enter the study. We cannot completely exclude the possibility that, for instance, differences in time of onset of symptoms and hospital admission causes modulation of inflammatory responses, for instance, cytokine responses from $\gamma \delta \mathrm{T}$ cells could have been missed due to their early response in infection, and may also play an important role in the host defence. Inferred from mouse models, respiratory inflammatory responses depend on the causative pathogen. We included CAP patients with different microbial aetiology. It would therefore be interesting to analyse larger cohorts of patients with viral or NP to investigate if Th17 responses are different from those found in pneumococcal pneumonia. Nevertheless, by studying a cohort of CAP patients independently of microbial aetiology, results can be easily translated to daily clinical practice.

In conclusion, our data indicate that CD4 T cells with a Th17 profile are engaged in local and systemic immune responses in CAP. Especially, our novel finding of significantly increased IL-17A ${ }^{+}$IL- $22^{+}$Th17 cells in BAL and peripheral blood supports their role in host defence in CAP.

\section{Competing interests None.}

Provenance and peer review Not commissioned; externally peer reviewed.

\section{REFERENCES}

1 van der Eerden MM, Vlaspolder F, de Graaff CS, et al. Comparison between pathogen directed antibiotic treatment and empirical broad spectrum antibiotic treatment in patients with community acquired pneumonia: a prospective randomised study. Thorax 2005;60:672-8.

2 Craig A, Mai J, Cai $S$, et al. Neutrophil recruitment to the lungs during bacterial pneumonia. Infect Immun 2009:77:568-75.
3 Marriott HM, Dockrell DH. The role of the macrophage in lung disease mediated by bacteria. Exp Lung Res 2007;33:493-505

4 Benito N, Moreno A, Miro JM, et al. Pulmonary infections in HIV-infected patients: an update in the 21st century. Eur Respir J 2012;39:730-45.

$5 O^{\prime}$ Connor W, Zenewicz LA, Flavell RA. Jr. The dual nature of $T(H) 17$ cells: shifting the focus to function. Nat Immunol 2010;11:471-6.

6 Park $H$, Li Z, Yang XO, et al. A distinct lineage of CD4 T cells regulates tissue inflammation by producing interleukin 17. Nat Immunol 2005;6:1133-41.

7 Aujla SJ, Chan YR, Zheng M, et al. IL-22 mediates mucosal host defense against Gram-negative bacterial pneumonia. Nat Med 2008;14:275-81.

8 Zenewicz LA, Flavell RA. IL-22 and inflammation: leukin' through a glass onion. Eur $J$ Immunol 2008;38:3265-8.

9 Lu YJ, Gross J, Bogaert D, et al. Interleukin-17A mediates acquired immunity to pneumococcal colonization. PLOS Pathog 2008:4:e1000159.

10 Olliver M, Hiew J, Mellroth P, et al. Human monocytes promote Th1 and Th17 responses to streptococcus pneumoniae. Infect Immun 2011;79:4210-17.

11 Zhang Z, Clarke TB, Weiser JN. Cellular effectors mediating Th17-dependent clearance of pneumococcal colonization in mice. J Clin Invest 2009;119:1899-909.

12 Happel KI, Dubin PJ, Zheng M, et al. Divergent roles of IL-23 and IL-12 in host defense against Klebsiella pneumoniae. J Exp Med 2005;202:761-9.

13 Ye P, Garvey PB, Zhang P, et al. Interleukin-17 and lung host defense against Klebsiella pneumoniae infection. Am J Respir Cell Mol Biol 2001;25:335-40.

14 Wu Q, Martin RJ, Rino JG, et al. IL-23-dependent IL-17 production is essential in neutrophil recruitment and activity in mouse lung defense against respiratory Mycoplasma pneumoniae infection. Microbes Infect 2007:9:78-86.

15 Fedele G, Nasso M, Spensieri F, et al. Lipopolysaccharides from Bordetella pertussis and Bordetella parapertussis differently modulate human dendritic cell functions resulting in divergent prevalence of Th17-polarized responses. J Immunol 2008:181:208-16.

16 Dubin PJ, Kolls JK. Pseudomonas aeruginosa and the host pulmonary immune response. Expert Rev Respir Med 2007;1:121-37.

17 Bermejo-Martin JF, Ortiz de Lejarazu R, Pumarola T, et al. Th1 and Th17 hypercytokinemia as early host response signature in severe pandemic influenza. Crit Care 2009;13:R201.

18 Hashimoto K, Durbin JE, Zhou W, et al. Respiratory syncytial virus infection in the absence of STAT 1 results in airway dysfunction, airway mucus, and augmented IL-17 levels. J Allergy Clin Immunol 2005;116:550-7.

19 Milner JD, Brenchley JM, Laurence $A$, et al. Impaired $T(H) 17$ cell differentiation in subjects with autosomal dominant hyper-IgE syndrome. Nature 2008;452:773-6.

20 British Thoracic Society Bronchoscopy Guidelines Committee a Subcommittee of Standards of Care Committee of British Thoracic Society. British Thoracic Society guidelines on diagnostic flexible bronchoscopy. Thorax 2001;56(Suppl 1):i1-21.

21 Ten Berge B, Paats MS, Bergen IM, et al. Increased IL-17A expression in granulomas and in circulating memory T cells in sarcoidosis. Rheumatology (Oxford) 2012:51:37-46.

22 Paats MS, Bergen IM, Hoogsteden HC, et al. Systemic CD4+ and CD8+ T cell cytokine profiles correlate with GOLD stage in stable COPD. Eur Respir J 2012:40:330-7.

23 Nembrini C, Marsland BJ, Kopf M. IL-17-producing T cells in lung immunity and inflammation. J Allergy Clin Immunol 2009;123:986-94; quiz 95-6.

24 Kemp K, Bruunsgaard H, Skinhoj P, et al. Pneumococcal infections in humans are associated with increased apoptosis and trafficking of type 1 cytokine-producing $T$ cells. Infect Immun 2002;70:5019-25.

25 Koh YY, Park Y, Lee HJ, et al. Levels of interleukin-2, interferon-gamma, and interleukin-4 in bronchoalveolar lavage fluid from patients with Mycoplasma pneumonia: implication of tendency toward increased immunoglobulin E production. Pediatrics 2001;107:E39.

26 Lee N, Wong CK, Chan PK, et al. Cytokine response patterns in severe pandemic 2009 H1N1 and seasonal influenza among hospitalized adults. PLoS One 2011;6: e26050.

27 Ouyang W, Kolls JK, Zheng Y. The biological functions of T helper 17 cell effector cytokines in inflammation. Immunity 2008;28:454-67.

28 Liang SC, Tan XY, Luxenberg DP, et al. Interleukin (IL)-22 and IL-17 are coexpressed by Th17 cells and cooperatively enhance expression of antimicrobial peptides. J Exp Med 2006:203:2271-9.

29 Sonnenberg GF, Nair MG, Kirn TJ, et al. Pathological versus protective functions of IL-22 in airway inflammation are regulated by IL-17A. J Exp Med 2010;207:1293-305.

30 Ye P, Rodriguez FH, Kanaly S, et al. Requirement of interleukin 17 receptor signaling for lung CXC chemokine and granulocyte colony-stimulating factor expression, neutrophil recruitment, and host defense. J Exp Med 2001;194:519-27.

31 Boutten A, Dehoux MS, Seta N, et al. Compartmentalized IL-8 and elastase release within the human lung in unilateral pneumonia. Am J Respir Crit Care Med 1996;153:336-42.

32 Monton C, Torres A, El-Ebiary M, et al. Cytokine expression in severe pneumonia: a bronchoalveolar lavage study. Crit Care Med 1999:27:1745-53. 